Supplementary Information on

\title{
Atomistic MD study of Nafion dispersions: Role of solvent and counter-ion in the aggregate structure, ionic clustering and acid dissociation
}

\author{
Atefeh Tarokh, Kunal Karan* and Sathish Ponnurangam* \\ Department of Chemical and Petroleum Engineering, University of Calgary \\ 2500 University Dr NW, Calgary, Alberta, Canada \\ (*kkaran@ucalgary.ca; *sathish.ponnurangam@ucalgary.ca)
}

\section{S1. Force field parameters, equilibration protocol, and validation details}

The form of force field used in this study is shown below. The parameters for individual bonded and nonbonded terms of Nafion macromolecule were obtained from ref. ${ }^{1}$. The parameters for solvents were adopted from $^{2,3,4}$.

$$
\begin{aligned}
E & =\sum_{\text {bond }}\left[K_{b 2}\left(b-b_{0}\right)^{2}+K_{b 3}\left(b-b_{0}\right)^{3}+K_{b 4}\left(b-b_{0}\right)^{4}\right] \\
& +\sum_{\text {angle }}\left[K_{a 2}\left(\theta-\theta_{0}\right)^{2}+K_{a 3}\left(\theta-\theta_{0}\right)^{3}+K_{a 4}\left(\theta-\theta_{0}\right)^{4}\right] \\
& +\sum_{\text {torsion }}\left[K_{t 1}(1-\cos (\varphi))+K_{t 2}(1-\cos 2 \varphi)+K_{t 3}(1-\cos 3 \varphi)\right] \\
& +\sum_{i j}\left\{\varepsilon_{i j}\left[2\left(\frac{\sigma_{i j}}{r_{i j}}\right)^{9}-3\left(\frac{\sigma_{i j}}{r_{i j}}\right)^{6}\right]+\frac{q_{i} q_{j}}{r_{i j}}\right\}
\end{aligned}
$$

In equation (S1) $b, \theta$ and $\varphi$ represent the internal coordinates of bond, angle and torsion angle, respectively and the subscript 0 represent their ground state positions. The $K_{b}, K_{a}$ and $K_{t}$ are the force constants for bond, angle, and torsion terms. 
The non-bond van der Waals (vdW) interactions are modeled using Lennard-Jones equation (LJ-9-6 form), where $\varepsilon_{i j}$ is the depth of the potential well, $\sigma_{i j}$ represents a finite distance where the inter-particle potential (potential between particle $i$ and $j$ ) is zero and $r$ is distance between the particles.

The following mixing rule is used for cross terms in LJ-9-6 equation:

$$
\begin{aligned}
& \sigma_{i j}=\left(\frac{\sigma_{i}^{6}+\sigma_{j}^{6}}{2}\right)^{1 / 6} \\
& \varepsilon_{i j}=2 \sqrt{\varepsilon_{i} \varepsilon_{j}} \frac{\sigma_{i}^{3} \sigma_{j}^{3}}{\sigma_{i}^{6}+\sigma_{j}^{6}}
\end{aligned}
$$

The Coulombic equation is used to calculate two-body electrostatic interaction, where $q_{i}$ represents partial charges of particle $i^{\text {th }}$.

\section{Validation of equilibration protocol}

Generally, the system and a property of interest Q (can be energetic, structural, or dynamical) is considered to have reached equilibrium, if the following two conditions are satisfied ${ }^{5}$,

$\boldsymbol{\tau}_{\text {equil }}>\boldsymbol{\tau}_{\text {relax }}(\mathrm{Q})$ and $\quad \boldsymbol{\tau}_{\text {sample }}>>\boldsymbol{\tau}_{\text {relax }}(\mathrm{Q})$

where, $\boldsymbol{\tau}_{\text {relax }}$ is the time by which trajectory average of the property Q does not fluctuate significantly $(<1 \%)$, $\boldsymbol{\tau}_{\text {equil }}, \boldsymbol{\tau}_{\text {sample }}$ are the equilibration and sampling times, respectively.

We have used the fluctuations in energetic and structural properties to verify the achievement of equilibrium state in our simulations. In particular, we have monitored fluctuations of total energy from its mean value (as a fraction of mean value, see equation below and Figure S1) as well as the stability of sulphur-sulphur RDF plots with time (Figures S2).

Fluctuation in energy, $\frac{\Delta \mathrm{E}}{\mathrm{E}}=\frac{\mathrm{E}(\mathrm{t})-\left|E_{\text {mean }}\right|}{\left|E_{\text {mean }}\right|}$ 
As seen in Figure S.1 and Table S1, the fluctuation in energy is very small $\left(\frac{\Delta E}{E}<1 \%\right)$ for all the cases. In particular, the energetic properties in all our simulation runs (except water and formamide) converged by $20 \mathrm{~ns}\left(\boldsymbol{\tau}_{\text {relax }}(\mathrm{Q})<20 \mathrm{~ns}\right)$. In the case of water and formamide, the properties converged by $110 \mathrm{~ns}$ and 50 ns, respectively. Whereas, structural properties such as sulfur-sulfur RDF converged within $100 \mathrm{~ns}$ for all systems. In addition, we also observed that the distances between ionic clusters reached a stable value by this time (as can be ascertained from Figure 6.b in the manuscript). Hence, our total simulation time of 250-300 ns is sufficient for the system and its properties to reach equilibrium.

a.

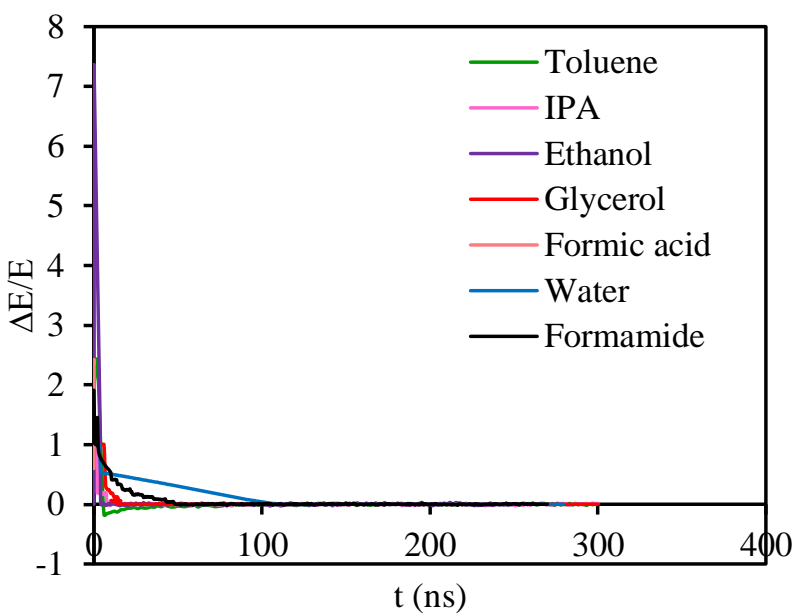

b.

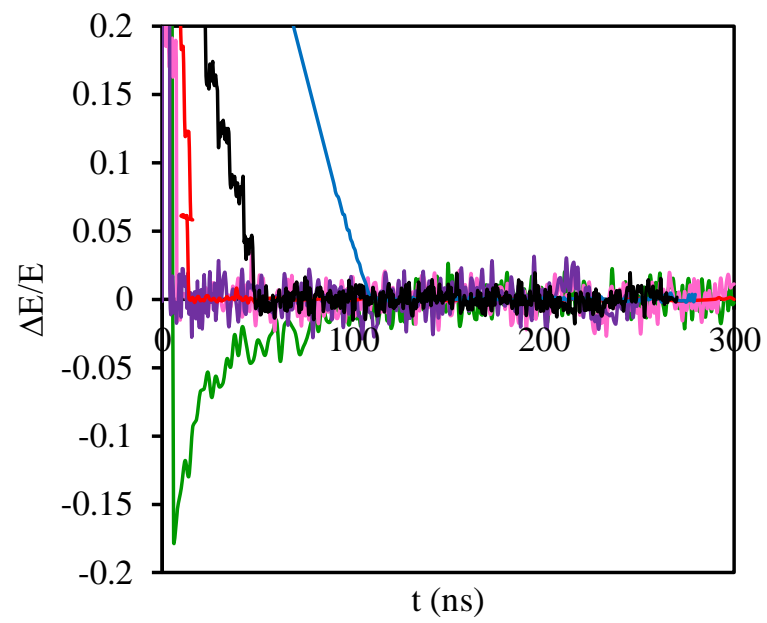

Figure S.1 (a) Energy fluctuation of the system during the run time, (b) is same as (a) but with expanded y-axis

Table S.1 Mean total energy and energy standard deviation.

\begin{tabular}{|c|c|c|}
\hline Solvent & $\begin{array}{c}\text { Average energy value } \\
\text { (kcal/mol) }\end{array}$ & $\begin{array}{c}\text { Standard deviation } \\
\text { (kcal/mol) }\end{array}$ \\
\hline Formamide & -24242.1 & 152.02 \\
\hline Water & -230864.7 & 264.1 \\
\hline Formic acid & -31368.8 & 128.04 \\
\hline Glycerol & -163716 & 135.9 \\
\hline Ethanol & -14965.2 & 174.5 \\
\hline IPA & -17889.3 & 183.5 \\
\hline Toluene & -16624.7 & 152.7 \\
\hline
\end{tabular}


a.

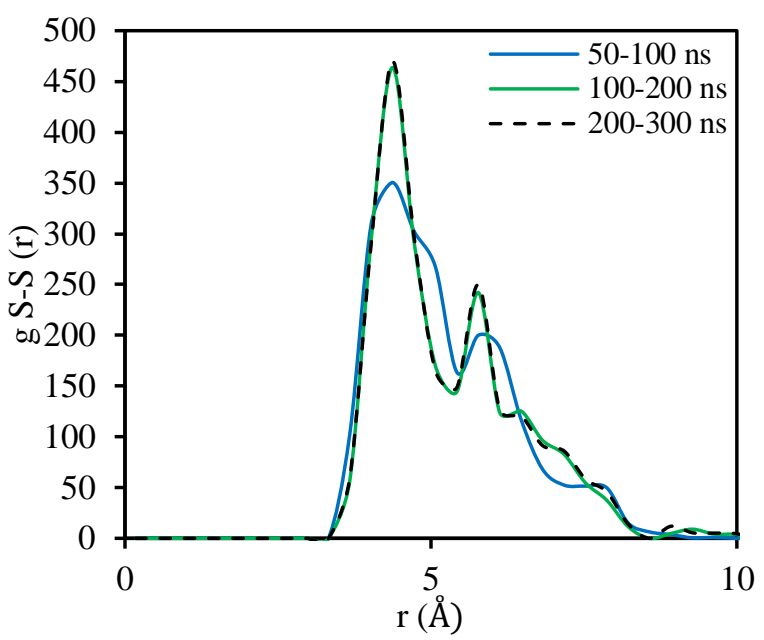

c.

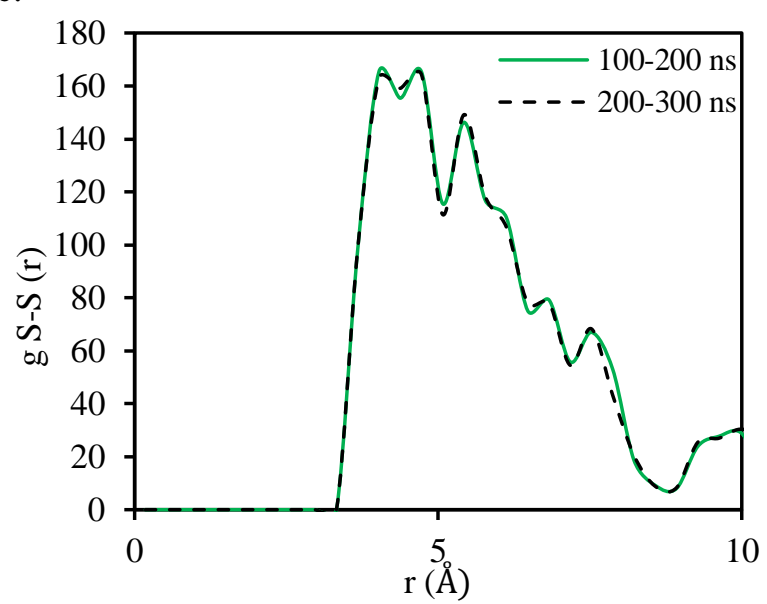

b.

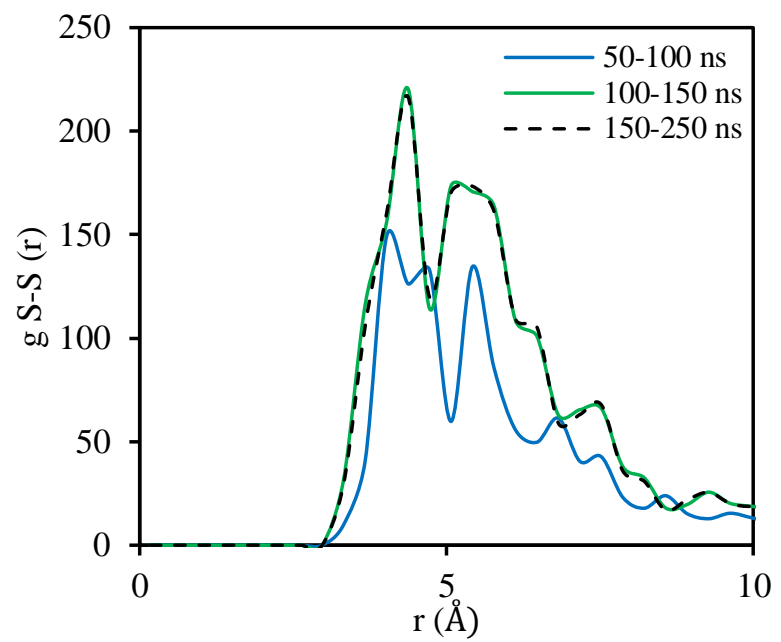

d.

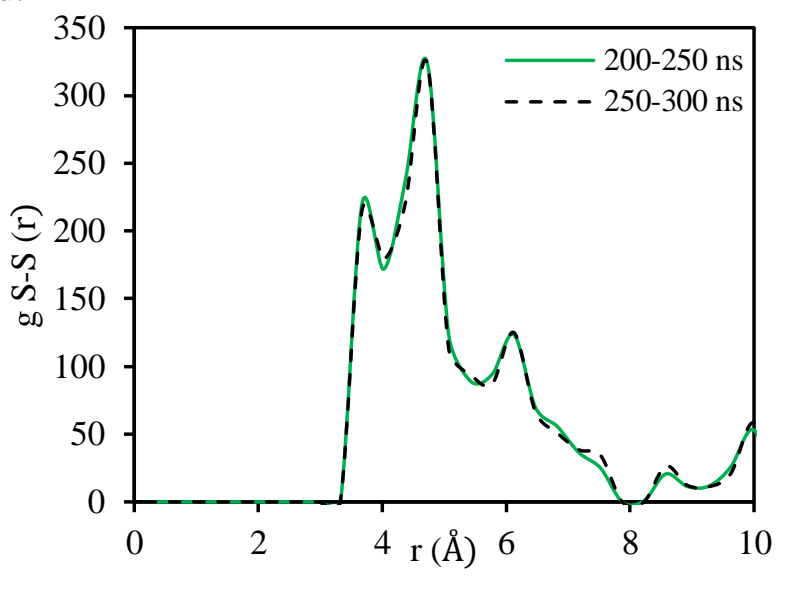

Figure S.2 Radial Distribution Function of sulphur-sulphur atom for one cluster during the run time a. Glycerol, b, Ethanol, c. IPA d. Toluene.

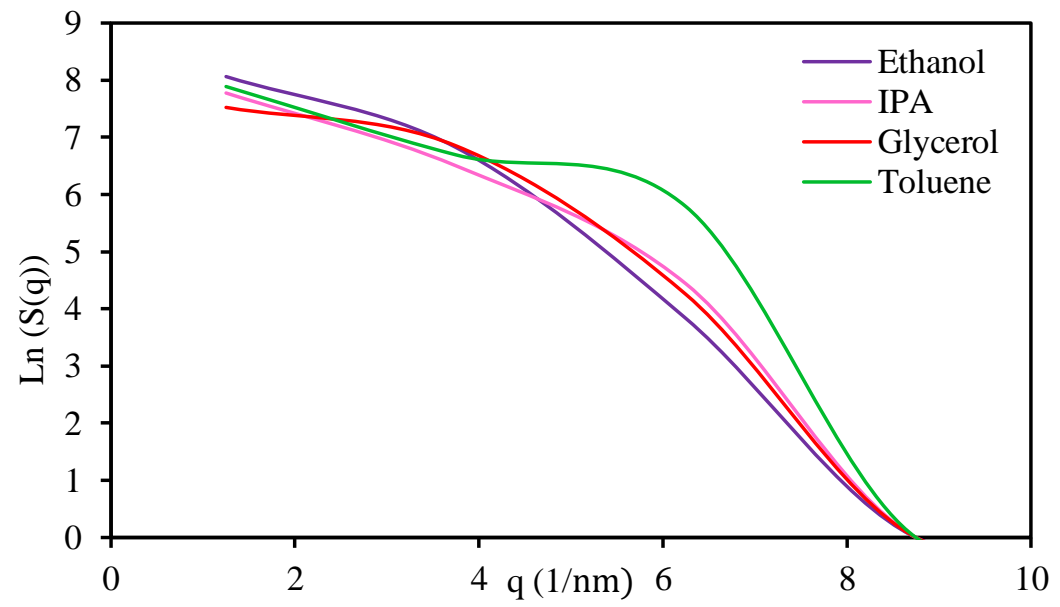

Figure S.3 $\ln [\mathrm{S}(\mathrm{q})]$ vs. q plot for ionic clusters of Nafion dispersions in different solvents 
a.

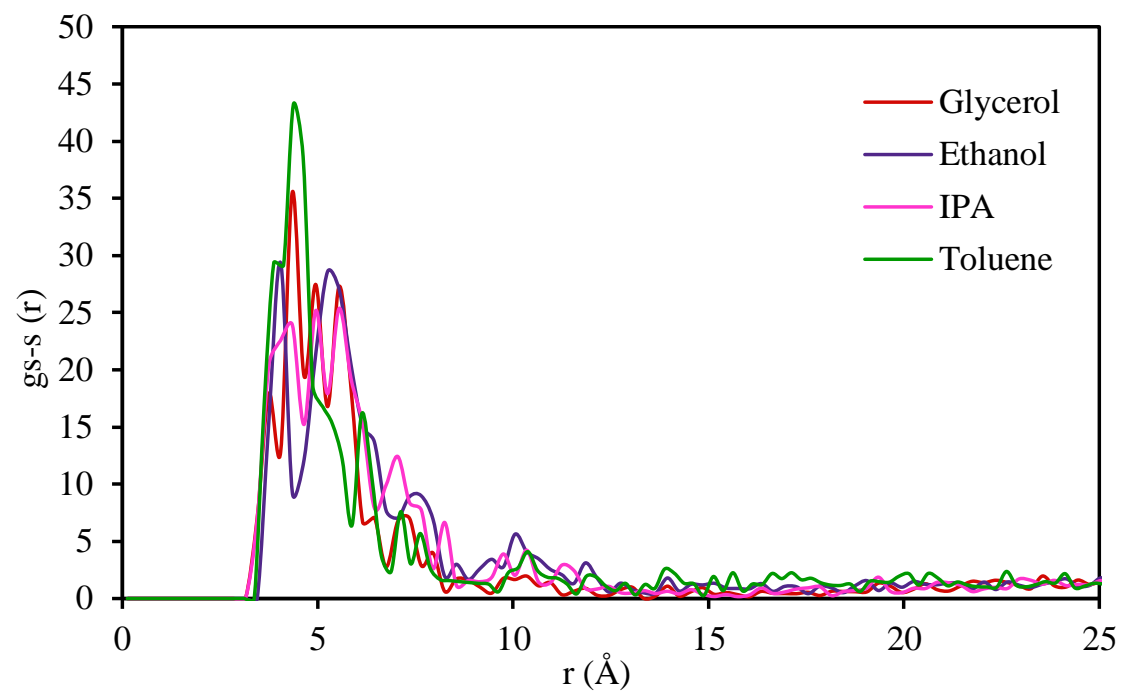

b.

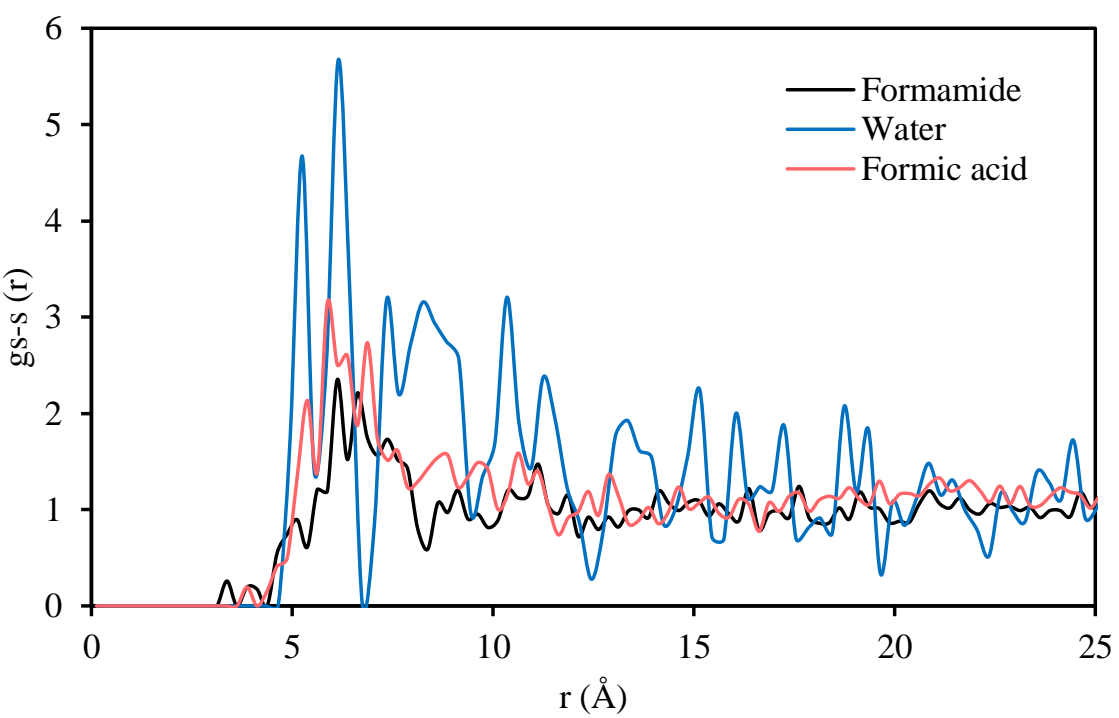

Figure S.4 Sulfur-Sulfur radial distribution function (RDF) of Nafion dispersions in $\mathbf{a}$. Low and medium dielectric constant solvents, b. High dielectric solvents. 


\section{S2 Effect of the backbone length}

We performed additional MD simulations with double the Nafion chain sizes for two of the dispersion systems (in IPA and glycerol) to verify if our findings regarding ionic clustering are still valid at higher MW of the polymers. We found that the presence of ionic clustering and the backbone aggregation (shape/size of aggregates and extent of swelling) were the same. Interestingly, the ionic cluster properties such as number of ion-pairs remained same as before (Figure 6a, S5, S6, \& S7). A comparison of RDFs of short and long chains for $\mathrm{S}-\mathrm{H}_{3} \mathrm{O}^{+}$and $\mathrm{S}-\mathrm{S}$ distances are shown in Figure.S6 and Figure.S7. The positions of the peaks in the RDF were the similar for both cases except for minor differences in the peak intensities. Visually, the Nafion chains can be observed to be more swollen in glycerol as compared to IPA (same trend was observed for shorter Nafion in respective solvents).

a.

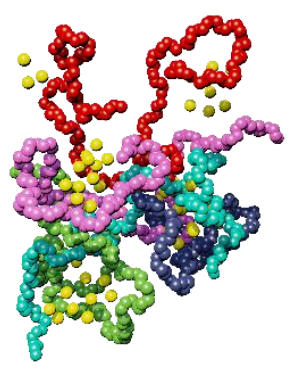

b.

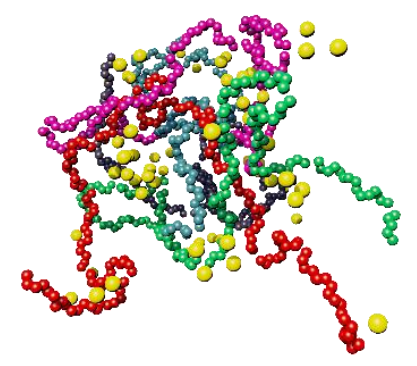

Figure S.5 long Nafion in a. IPA. b. Glycerol. 
a.

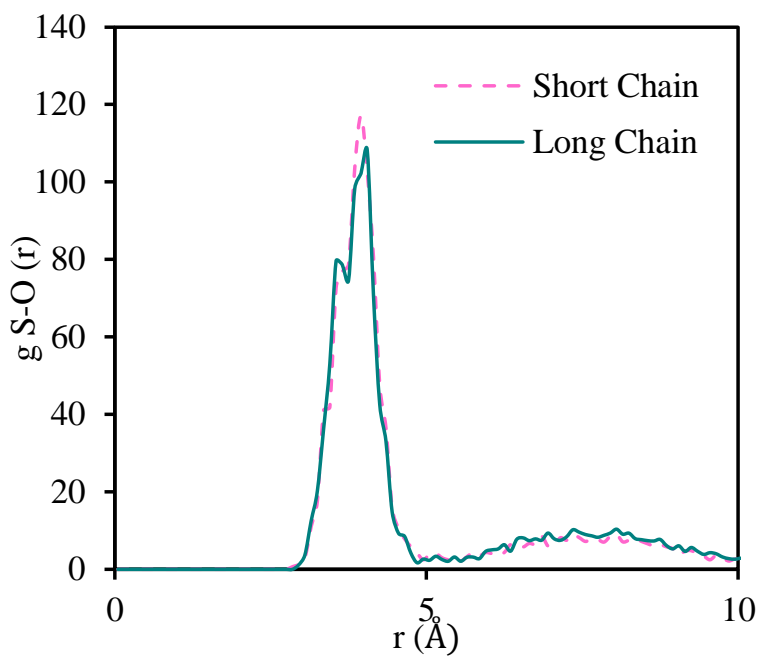

b.

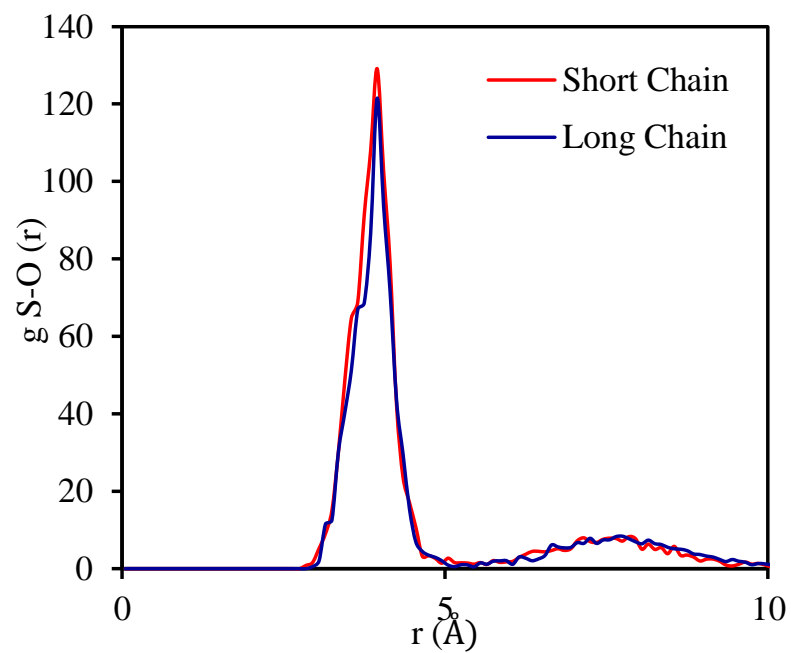

Figure S.6 Sulfur- Hydronium (oxygen) radial distribution function a. IPA, b. Glycerol.

a.

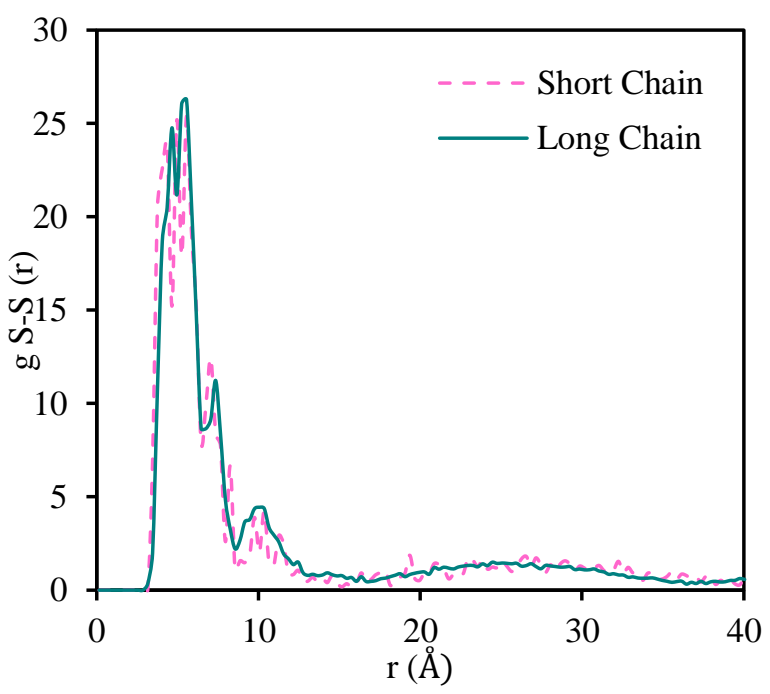

b.

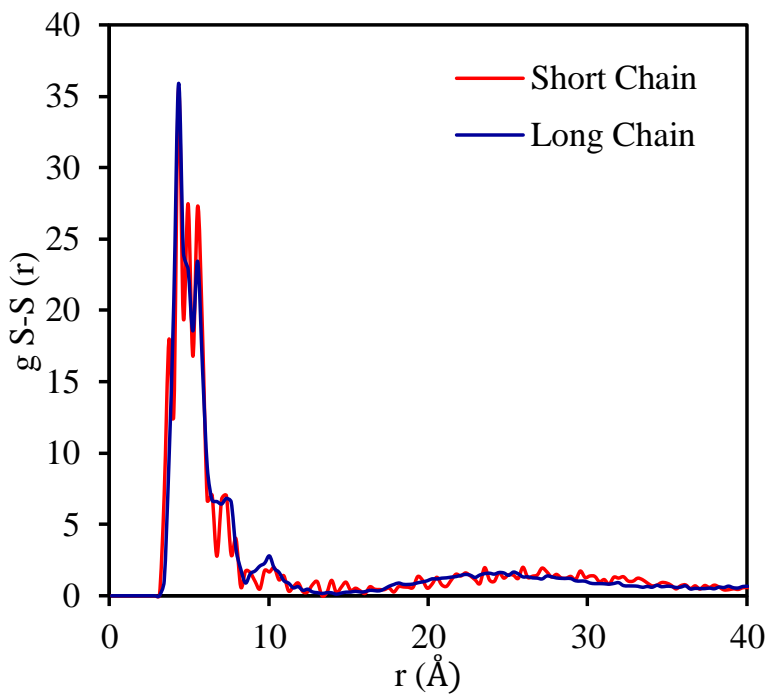

Figure S.7 Sulfur-Sulfur radial distribution function a. IPA, b. Glycerol

\section{S3 Effect of counterion on Nafion aggregation in water/IPA system}

As shown in Figure 12, in the main text, we studied the dispersion of Nafion in a 50/50 wt $\%$ water/IPA system. Herein, we modeled the mentioned system with two different counterions, hydronium, and sodium ions. As discussed in the paper, the counterion type has a strong impact on the Nafion aggregation in dispersion systems. The following figures illustrate the radial distribution function (RDF) of sulfurcounterion and sulfur- sulfur in these two systems. According to the sulfur- counterion RDF (Figure S.8 a), 
the sodium ions have a stronger attraction to the sulfur atoms in the side chains in comparison with the hydronium molecules. In other words, when the counterion of the system is hydronium ion, the counterions are dispersed in the dispersion system. However, according to the sulfur-sulfur RDFs (Figure S.8 b), there are no sulfonic acid clusters in both systems.

a.

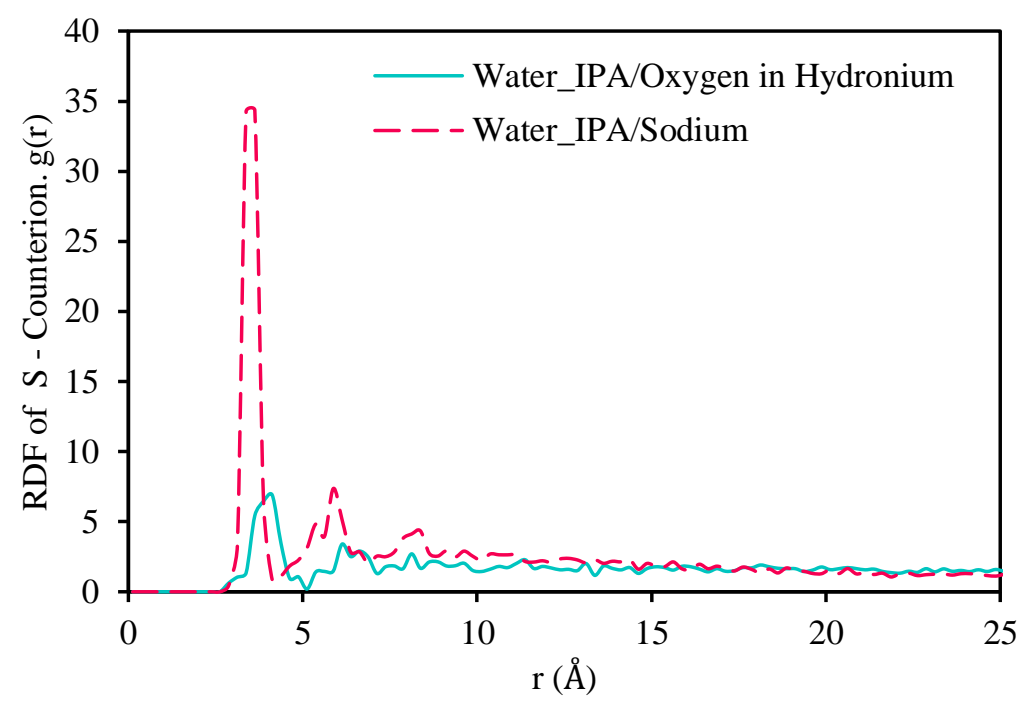

b.

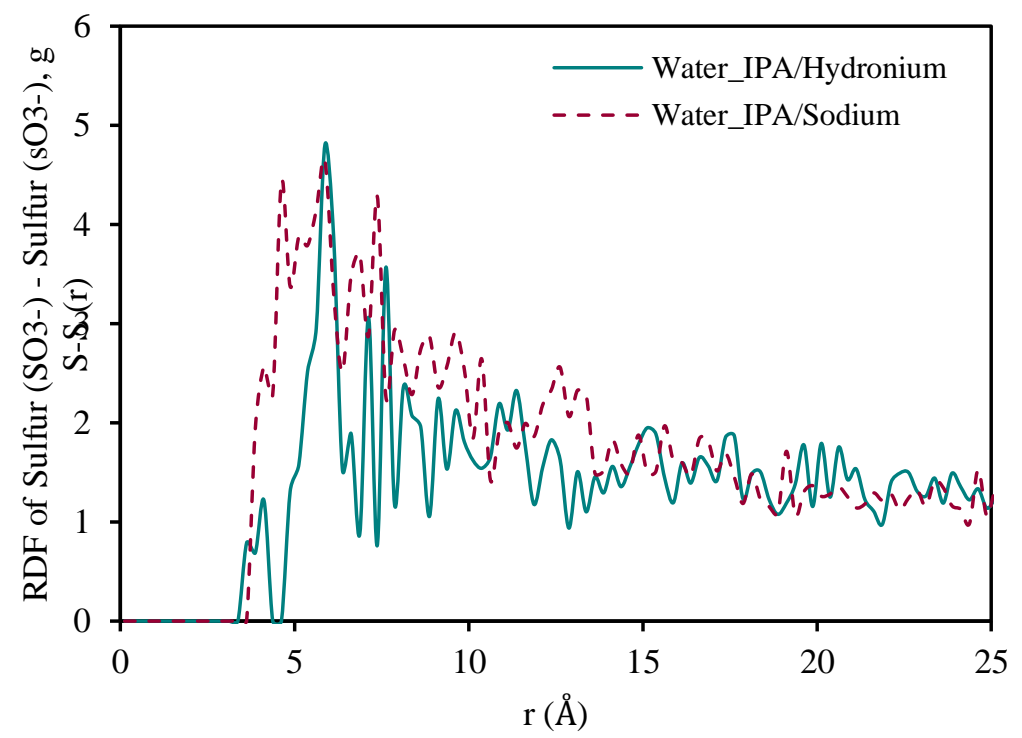

Figure S.8 a. Sulfur-counterion radial distribution function (RDF) of Nafion dispersions in water/IPA mixture with hydronium and sodium ion as the counterions. b. Sulfur-sulfur radial distribution function (RDF) of Nafion dispersions in water/IPA mixture with hydronium and sodium ion as the counterions 


\section{References}

(1) Jinnouchi, R.; Kudo, K.; Kitano, N.; Morimoto, Y. Molecular Dynamics Simulations on O2 Permeation through Nafion Ionomer on Platinum Surface. Electrochim. Acta 2016, 188, 767-776. https://doi.org/10.1016/j.electacta.2015.12.031.

(2) Pérez, M. LAMMPS User Manual; 2016. https://doi.org/10.1002/ejoc.201200111.

(3) Sun, H. COMPASS: An Ab Initio Force-Field Optimized for Condensed-Phase ApplicationssOverview with Details on Alkane and Benzene Compounds. J. Phys. Chem. B 1998, 5647, 7338-7364. https://doi.org/10.1021/jp980939v.

(4) Rigby, D.; Sun, H.; Eichinger, B. E. Computer Simulations of Poly(Ethylene Oxide): Force Field, PVT Diagram and Cyclization Behaviour. Polym. Int. 1997, 44, 311-330.

(5) van Gunsteren, W. F.; Mark, A. E. Validation of Molecular Dynamics Simulation. J. Chem. Phys. 1998, 108 (15), 6109-6116. https://doi.org/10.1063/1.476021. 